\title{
Consideraciones para el almacenamiento de archivos digitales en la nube informática en bibliotecas universitarias
}

\author{
Brenda Cabral Vargas*
}

Artículo recibido:

26 de enero de 2016

Artículo aceptado:

27 de septiembre de 2016

\section{Resumen}

El presente trabajo tiene por objeto identificar y describir algunos aspectos en torno al almacenamiento de archivos digitales en la nube. Para ello partimos de definir qué es la nube, para luego describir sus características. Además se señalan algunas ventajas, desventajas y limitaciones que tiene el almacenamiento en la nube informática, así como recomendaciones para las instituciones de educación superior que requieran planear un proyecto de arquitectura de nube para preservar sus archivos digitales. Se consideran únicamente los aspectos de planeación, no aquellos relacionados con la gestión u organización documental en la nube.

* Instituto de Investigaciones Bibliotecológicas y de la Información, UNAM brenda@iibi.unam mx

INVESTIGACIÓN BIBLIOTECOLÓGICA, vol.32, núm.74, enero/marzo, 2018, México, ISSN: 2448-8321. pp. 55-75 
Palabras clave: Archivos Digitales; Nube Informática; Documentos para Bibliotecas Universitarias; Contenidos Digitales

\section{Requirements of digital file storage in the cloud for} academic libraries

Brenda Cabral-Vargas

\section{Abstract}

This paper identifies and describes several aspects associated with storage of digital files in the cloud. A definition of the cloud is ventured and its major features detailed in order to distinguish advantages, disadvantages and limitations that must be taken into account when information is stored in the cloud. Several recommendations are provided for higher education institutions tasked with planning the architecture to be deployed for organizing digital files using the cloud computing.

Keywords: Digital Files; Cloud Computing; Documents for Academic Libraries; Digital Content

\section{INTRODUCCIÓN}

Con el tiempo, a la educación superior se le ha reconocido como uno de los pilares del desarrollo de la sociedad, en cuya transformación han contribuido las universidades a través de los vínculos entre el gobierno y la industria, los investigadores y los estudiantes. La tendencia observada durante los últimos años es la transición de las universidades en un proceso de actualización continua dentro de lo que se conoce como TI (tecnología de la información), como base para las actividades educativas y de investigación.

Con la evolución de la tecnología, el número de servicios que migran de lo tradicional a lo digital crece día con día. Para que estos servicios se proporcionen en una forma adecuada deben encontrarse en el entorno digital, utilizando las tecnologías apropiadas y garantizando el acceso rápido y fácil a una gran cantidad de usuarios. Con base en esto las unidades de información implementan servicios enmarcados dentro del contexto de las TI, tomando siempre en consideración los aspectos referentes al costo-beneficio. 
Debido a la situación económica de nuestro país, en este momento las universidades enfrentan un dramático aumento de costos en el proceso de educación y la disminución del presupuesto asignado, lo que conduce a la presión por encontrar medios alternativos para alcanzar su objetivo, que es el de formar estudiantes y llevar a cabo la investigación. En esta situación, las unidades de información son por lo común las primeras en la lista de recortes presupuestales, lo que provoca desabasto de información y de mecanismos para facilitar su flujo.

Como respuesta a estas presiones, las universidades también deben realizar cambios con el fin de reorientar sus servicios para optimizar con eficiencia y eficacia tanto las operaciones internas como las interacciones con los principales grupos de interés. Por su parte, las unidades de información deben orientar sus servicios y llevar a cabo diversas actividades para preparar al personal que ahí labora y a los usuarios de la misma, y de esta manera contar con las características para ser incluidas en el proceso de reajuste institucional (en términos de estrategia, estructura, cultura y procesos), pero también en lo referente a la tecnología (de acuerdo con la semántica, las aplicaciones, la arquitectura y la infraestructura de las instituciones modernas).

A nivel de organización, la nube computacional (cloud computing) se puede considerar una alternativa de utilización de las TI para el entorno educativo, sobre todo en las condiciones actuales de carencia de recursos financieros.

En el presente, hemos llegado a un punto en el que la nube está entre nosotros de forma definitiva e irreversible. Hace no mucho tiempo que no conocíamos otro servicio de almacenamiento de archivos diferente a Dropbox, y ahora hay tantos, provistos por empresas tan importantes como Google, que cuesta trabajo decidirse por el uso de uno u otro. Y lo más interesante es que para un usuario normal esta nube se puede aprovechar al máximo y con ello dejar de depender casi totalmente del almacenamiento local.

Desde este punto de vista, es importante identificar los datos, servicios y procesos que son candidatos adecuados para residir en la nube, dentro de un proceso de planeación para que la migración tenga sentido desde el punto de vista financiero.

El potencial y la eficiencia de uso de la nube informática en la educación superior han sido reconocidos por muchas universidades debido a que ofrecen la posibilidad de concentrarse en las actividades de enseñanza e investigación, y permiten a las unidades de información realizar sus procesos de captación, organización y resguardo y ofrecer servicios de información acordes con las necesidades de las universidades. Además, éstas pueden emplear las soluciones de la nube para apoyar, en el aspecto académico, el aprendizaje cooperativo y las teorías de aprendizaje social orientado, utilizando 
tecnologías informáticas para dar soporte a los métodos de colaboración de la instrucción, y por otra parte dar salida más ágil a sus unidades de información para ofrecer servicios de calidad, permitiendo trabajar con otras unidades para ampliar su campo de acción e interactuando para dar soporte a proyectos como el e-learning, proporcionando un servicio de información acorde con la infraestructura, la plataforma y los servicios educativos.

\section{QUÉ ES LA NUBE INFORMÁTICA}

Es importante resaltar que el término nube informática ha causado confusión a muchas personas. La palabra "nube" se utiliza como una metáfora de internet, su principal objetivo es la personalización y se define por la experiencia del usuario. En otras palabras, los recursos de computación en nube proporcionan información y software compartido a través de internet con un sistema de pago por servicio (pay-as-you-go).

El término nube se origina en el mundo de las telecomunicaciones en 1960, cuando los proveedores empezaron a utilizar los servicios de red privada virtual o VPN para las comunicaciones de datos (Kaufman, 2009). Un hecho curioso con respecto a este concepto: John McCarthy opinó en 1960 que el concepto de la computación en nube algún día podría ser organizado como un servicio público.

Nube se comenzó a utilizar con las características que conocemos hoy en día desde el 2006, por proveedores de servicios de internet como Google, Amazon y Microsoft, que habían conseguido construir en sus organizaciones un sistema de recursos distribuidos de manera horizontal, introducidos como servicios virtuales de la tecnología de la información, escalados masivamente y manejados como recursos configurados y asociados de manera continua.

La nube informática también se conoce con otros términos como servicios en la nube, informática en la nube, nube de cómputo o nube de conceptos, del inglés cloud computing.

El Laboratorio de Tecnologías de la Información, integrado en el National Institute of Standards and Technology (NIST) del Departamento de Comercio del Gobierno Federal de los Estados Unidos, ha definido nube informática de la siguiente forma:

Cloud Computing es un modelo que permite el acceso bajo demanda y a través de la red a un conjunto de recursos compartidos y configurables (como redes, servidores, capacidad de almacenamiento, aplicaciones y servicios) que pueden ser rápidamente asignados y liberados con una mínima gestión por parte del proveedor del servicio. (NIST, 2011) 
En otras palabras, la nube informática o la computación en la nube, como la conocen otros autores, se refiere a servidores desde internet encargados de atender las peticiones en cualquier momento. Se puede tener acceso a su información o servicios mediante una conexión a internet desde cualquier dispositivo móvil o fijo ubicado en cualquier lugar. Sirven a sus usuarios desde varios proveedores de alojamiento repartidos frecuentemente también por todo el mundo. Esta medida reduce los costos, garantiza un mejor tiempo de utilización y que los sitios web sean seguros para evi tar a los hackers u otros actores que pudieran querer modificar o afectar la información almacenada en la nube.

Según el NIST, el modelo tiene las cinco características esenciales siguientes:

1. Autoservicio bajo demanda. El usuario puede acceder a capacidades de computación en la nube de forma automática a medida que las vaya requiriendo sin necesidad de una interacción humana con su proveedor o sus proveedores de servicios de nube.

2. Múltiples formas de acceder a la red. Los recursos son accesibles a través de la red y por medio de mecanismos estándar que son utilizados por una amplia variedad de dispositivos de usuario, desde teléfonos móviles a ordenadores portátiles o PDA. ${ }^{1}$

3. Compartición de recursos. Los recursos (almacenamiento, memoria, ancho de banda, capacidad de procesamiento, máquinas virtuales, etc.) de los proveedores son compartidos por múltiples usuarios, a los que se van asignando capacidades de forma dinámica según sus peticiones. Los usuarios pueden ignorar el origen y la ubicación de los recursos a los que acceden, aunque sí es posible que sean conscientes de su situación a determinado nivel, como el de CPD (centro de procesamiento de datos) o el de país.

4. Elasticidad. Los recursos se asignan y liberan rápidamente, muchas veces de forma automática, lo que da al usuario la impresión de que los recursos a su alcance son ilimitados y están siempre disponibles.

5. Servicio medido. El proveedor es capaz de medir, a determinado nivel, el servicio efectivamente entregado a cada usuario, de forma que tanto proveedor como usuario tienen acceso transparente al consumo real de los recursos, lo que posibilita el pago por el uso efectivo de los servicios (NIST, 2011).

1 PDA viene del inglés personal digital assistant, asistente digital personal, también conocido como computadora de bolsillo, organizador personal o agenda electrónica de bolsillo. Es una computadora de mano originalmente diseñada como agenda electrónica con la finalidad de utilizarla para organizar datos (incluye calendario, lista de contactos, bloc de notas, recordatorios, programa de dibujo, etc.). 
Hay muchas prácticas y ejemplos sobre el uso de la nube informática. Esto permitió a las instituciones tanto públicas como privadas reducir los gastos de TI (mediante la reducción de las necesidades de la concesión de licencias y la actualización del software), mantener su propia organización de datos, así como mejorar los recursos de TI para las investigaciones y los estudiantes.

Sin embargo, un estudio reciente con respecto al uso de la nube informática en la educación superior muestra que las universidades todavía se pueden encontrar en la fase de "adopción" junto a otros sectores, como el comercial y gubernamental (Katz, Goldstein y Yanosky, 2010). Los factores importantes que influyen en la decisión de utilizar la nube informática en la educación superior difieren un poco de los otros sectores. Así, según Katz, Goldstein y Yanosky (2010), el 70 \% de los líderes de TI de la educación superior (de 302 encuestados) han considerado que la mejora de los servicios de TI es el factor de decisión más importante, mientras que sólo el 38 \% dio la misma importancia a la reducción de costos.

El uso de la nube informática en la educación superior debe ser analizado tanto desde el punto de vista de los beneficios como de los riesgos y limitaciones (Tabla 1). Aún con las desventajas potenciales que presentan, la computación en la nube y las aplicaciones web van ganando más espacio. Lo anterior deriva de que las empresas que ofrecen estos servicios buscan las maneras de maximizar las ventajas y reducir las desventajas haciendo que la computación en la nube tenga cada vez más fuerza en la World Wide Web.

\begin{tabular}{|l|l|}
\hline \multicolumn{1}{|c|}{ Beneficios } & \multicolumn{1}{|c|}{ Limitaciones } \\
\hline $\begin{array}{l}\text { El acceso a las aplicaciones desde cualquier } \\
\text { lugar }\end{array}$ & No todas las aplicaciones "corren" en la nube \\
\hline $\begin{array}{l}\text { Apoyo a la enseñanza y el aprendizaje, facilita } \\
\text { flujo de información }\end{array}$ & $\begin{array}{l}\text { Existen riesgos relacionados con la protección } \\
\text { de datos y seguridad }\end{array}$ \\
\hline $\begin{array}{l}\text { Se puede emplear software gratuito o de pago } \\
\text { por uso }\end{array}$ & $\begin{array}{l}\text { Se requiere apoyo organizacional para su } \\
\text { aplicación }\end{array}$ \\
\hline $\begin{array}{l}\text { La información puede ser consultada durante } \\
\text { las 24 horas los 365 días del año }\end{array}$ & $\begin{array}{l}\text { La circulación de la información se puede limi- } \\
\text { tar por política institucional y por la propiedad } \\
\text { intelectual }\end{array}$ \\
\hline $\begin{array}{l}\text { Apertura al entorno empresarial y de investi- } \\
\text { gación avanzada, facilita las colaboraciones } \\
\text { institucionales }\end{array}$ & \begin{tabular}{l} 
La seguridad y protección de datos es sensible \\
\hline
\end{tabular} \\
\hline
\end{tabular}




\begin{tabular}{|c|c|}
\hline $\begin{array}{l}\text { Protección del medio ambiente mediante el uso } \\
\text { de tecnologías verdes }{ }^{2}\end{array}$ & $\begin{array}{l}\text { Vencimiento de soluciones. El software puede } \\
\text { no ser adecuado al servicio de información }\end{array}$ \\
\hline $\begin{array}{l}\text { Presenta mayor apertura a la comunidad } \\
\text { académica hacia las nuevas tecnologías }\end{array}$ & Provoca desconfianza en algunas personas \\
\hline Aumento de capacidades funcionales & Normas de adhesión para el uso \\
\hline $\begin{array}{l}\text { Uso sin conexión, lo que facilita sincronización, } \\
\text { permitiendo el intercambio de información }\end{array}$ & $\begin{array}{l}\text { La velocidad/falta de internet puede afectar los } \\
\text { métodos de trabajo }\end{array}$ \\
\hline
\end{tabular}

Tabla 1. Beneficios y limitaciones del uso de la nube

Fuente: adaptación propia basada en Mircea y Andreescu (2011)

Conviene mencionar, además, las desventajas evidentes, entre las que tenemos:

- Percepción de pérdida de privacidad de datos sensibles.

- Dependencia de la infraestructura y plataformas de terceros para operar.

- Nuestra información queda vulnerable a robo o hackeo.

- La madurez funcional de las aplicaciones hace que continuamente estén variando sus interfaces, por lo cual el aprendizaje en instituciones de orientación no tecnológica puede causar dependencias con el servicio.

- Sin internet no tendremos acceso a nuestra información.

Las desventajas pueden ser minimizadas si se analizan y evalúan las características de aquellas empresas que ofrecen servicios de nube informática, es decir, qué las hace sobresalir de las demás, qué beneficios ofrecen, así como sus garantías, su solvencia y solidez en el mercado. De esta forma se puede estar más seguro de que los datos estarán protegidos de manera adecuada y de que los usuarios (alumnos, académicos y administrativos) podrán acceder a los servicios sin ningún tipo de problema.

Después del análisis, se pueden seleccionar uno o más modelos de nube informática para utilizar. La decisión depende de las necesidades reales y éstas se pueden alinear con la estrategia de flujo de información de la universidad. Por consiguiente, las unidades de información deben de ser elegidas dentro de este proceso para integrarse y hacer uso de este servicio y estar en sintonía para apoyar los objetivos de la universidad.

2 Tecnología verde es el uso eficiente de los recursos computacionales minimizando el impacto en el ambiente (ahorro de energía), maximiza su viabilidad económica y asegura deberes sociales. 
Como todo proyecto, puede ser que para algunas unidades de información los gastos de inversión para iniciar resulten onerosos, pero a mediano plazo se puede compensar lo invertido haciendo posible la reducción en el gasto de uso de hardware, la generación de copias de seguridad y su almacenamiento, sincronización y recuperación de información, la facilidad en el flujo de la misma, entre otras acciones, con lo cual se puede justificar dicha inversión. De igual forma, también se pueden optimizar recursos mediante el empleo de software de acceso abierto, con lo que la operación de uso puede bajar costos.

Para tomar una decisión acerca de cómo emplear la nube informática como un soporte de información, se debe considerar el riesgo de implementación asociada con la solución, esto es, mediante la toma de decisiones para la solución, la cual debe brindar una ganancia que supere los costos invertidos. También es importante hacer un análisis general para determinar qué riesgos específicos en relación con la nube pueden ser transferidos a los proveedores. Dentro de este análisis también se deben tener en cuenta los riesgos a mediano plazo en caso de no emplear esta tecnología.

En cuanto a los riesgos de implementación, las principales preocupaciones acerca de la adopción en las unidades de información son la seguridad, el rendimiento y la disponibilidad. Como se mostró en la Tabla 1, existen diversos beneficios y limitantes que deben ser considerados para implementar este servicio; no obstante, existen otros beneficios de carácter específico que pueden apuntalar la información en el momento de justificar el proyecto.

Para el caso de una unidad de información esto redunda en que el almacenamiento de datos en la nube garantiza que nunca habrá escasez de espacio para la información. Una unidad de información puede comenzar con un servidor conectado a la nube para el procesamiento de la información. Por otra parte, tan pronto como el sitio web comienza a ser un gran nudo de tráfico o si se agregan más bases de datos, el propietario del sitio puede aumentar la capacidad del procesador o redireccionar para agilizar el flujo de información. Esta flexibilidad es ofrecida por los servicios de administración de la nube, lo que da como resultado una alternativa de solución viable.

A diferencia de lo que muchos creen, el uso virtual de la información no reduce el ámbito del hardware, considerando que el equipo tiene un disco duro físico, el cual tiene un rango limitado de capacidad; por el contrario, el uso virtual de información permite a cada usuario crear dos unidades virtuales, de manera que la presión de flujo de información se puede distribuir correctamente. En cuanto a los recursos, casi no hay diferencia entre la unidad 
de disco duro física y virtual. El disco duro virtual puede tener CPU, RAM, almacenamiento y capacidades de redes, igual a la capacidad de poder de un disco duro físico.

Un servidor virtual puede tener propiedades similares a las del servidor regular, tales como acceso de administrador, supervisión y otras características relacionadas. La nube informática se basa en la tecnología de uso virtual de la información de servidores, porque los equipos cliente se conectan con diferentes servidores, muchos de los cuales son virtuales. El servidor virtual ofrece instalaciones de almacenamiento en la nube y, por tanto, las unidades de información pueden almacenar sus datos.

\section{Arquitectura de la NUbe PARA SER IMPLEMENTADA POR LAS UNIVERSIDADES}

Toda aquella institución que desee implantar un proyecto de este tipo debe pensar en planificar y trabajar en los requerimientos de la nube para hacer frente a retos específicos y a su vez definir qué es lo que se requiere, a fin de desvanecer la incertidumbre con respecto a problemas que se puedan presentar durante el desarrollo del proyecto, como podría ser la privacidad, el riesgo de no obtener el rendimiento propuesto, la interoperabilidad, la capacidad, la disponibilidad de la red, el personal y las percepciones, entre otras.

La adopción de la arquitectura para incursionar en la nube informática implica la superación de barreras, tales como cuestiones de política interna y de control, los nuevos servicios que se brindarán que podrían quedar por encima de los ya existentes, incluir al personal de todas las edades para hacer uso de estos servicios, y emprender estrategias para formar entornos a la elección del consumidor (Katz, Goldstein y Yanosky, 2010).

En la arquitectura que se utiliza en la nube para almacenar información, los datos residen sobre todo en servidores localizados en algún sitio de internet y la aplicación se ejecuta tanto en los servidores de la nube (cloudservert) como en el navegador del usuario; por ejemplo, cuando utilizamos Gmail, Google Maps, servicios de Yahoo o muchos de los servicios de eBay, ${ }^{3}$ estamos usando esta arquitectura. La Figura 1 representa visualmente cómo se dan las relaciones dentro de una nube en cuanto a los elementos que intervienen en ella.

3 Es un sitio destinado a la subasta de productos a través de internet. Fundado en 1995, es uno de los pioneros en este tipo de transacciones. Desde 2002 eBay es propietario de PayPal. Desde 2015 su director ejecutivo (CEO) en Devin Wenig. 


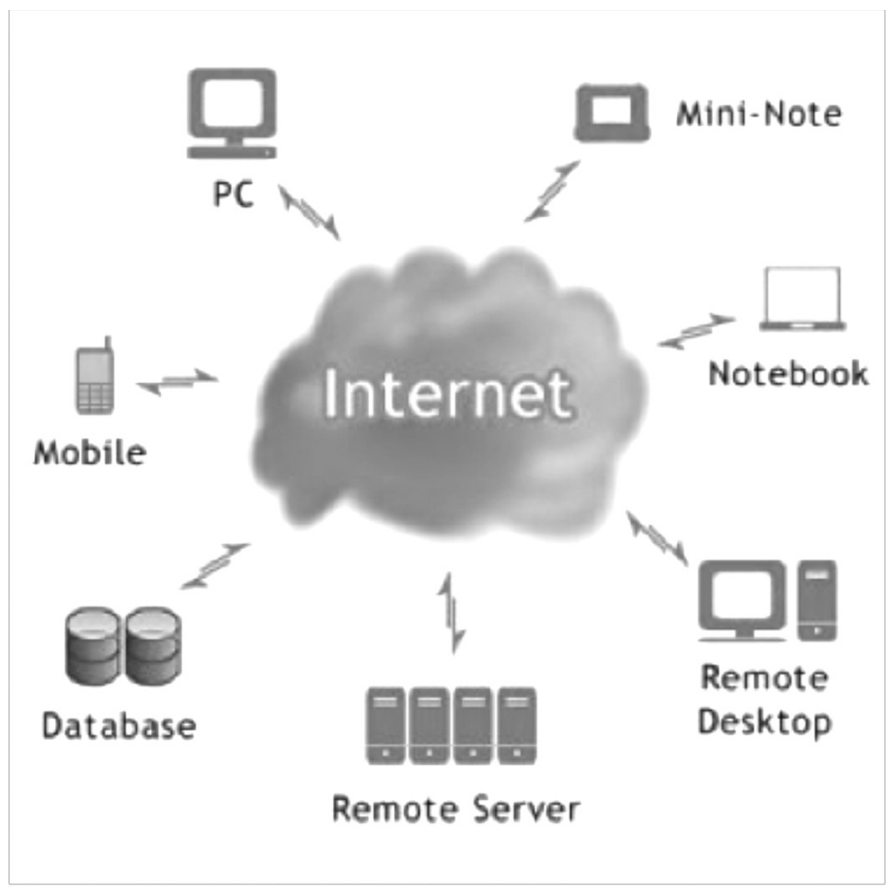

Figura 1. Relación de elementos en la nube Fuente: Cuadros (2013)

El patrón arquitectónico donde se ubicarían las unidades de información en la nube informática dentro de las universidades se puede describir a partir del desarrollo y suministro de los servicios que se ofrecerán como apoyo en el proceso de formación (Figura 2).

Es importante resaltar que el modelo de nube comunitaria apareció debido al aumento de la presión en el ámbito educativo (necesidad de la elaboración de informes, seguimiento informativo, demográfico y financiero que comienza desde el momento de inscribir a los estudiantes y hasta el final de la etapa educativa) y también debido a las ventajas aprovechadas de la colaboración (la evaluación del éxito en el mercado laboral, haciendo hincapié en la calidad de la educación, la innovación), así como para consultar los servicio en las unidades de información.

Para el caso de las unidades de información, ésta se administra de acuerdo con la organización de sus acervos y con los servicios de información que ofrezcan de manera centralizada. La creación de informes y análisis de tendencias dará lugar a decisiones bien fundadas con respecto a los servicios 
que se deben ofrecer, incluyendo el desarrollo de colecciones o la creación y/o la cancelación de servicios, con base en necesidades identificadas. El proceso siguiente ilustra el método en el que las unidades de información pueden acceder y modificar la información de una nube comunitaria (Figura 3).

\section{Arquitectura de la Nube para la Universidad}

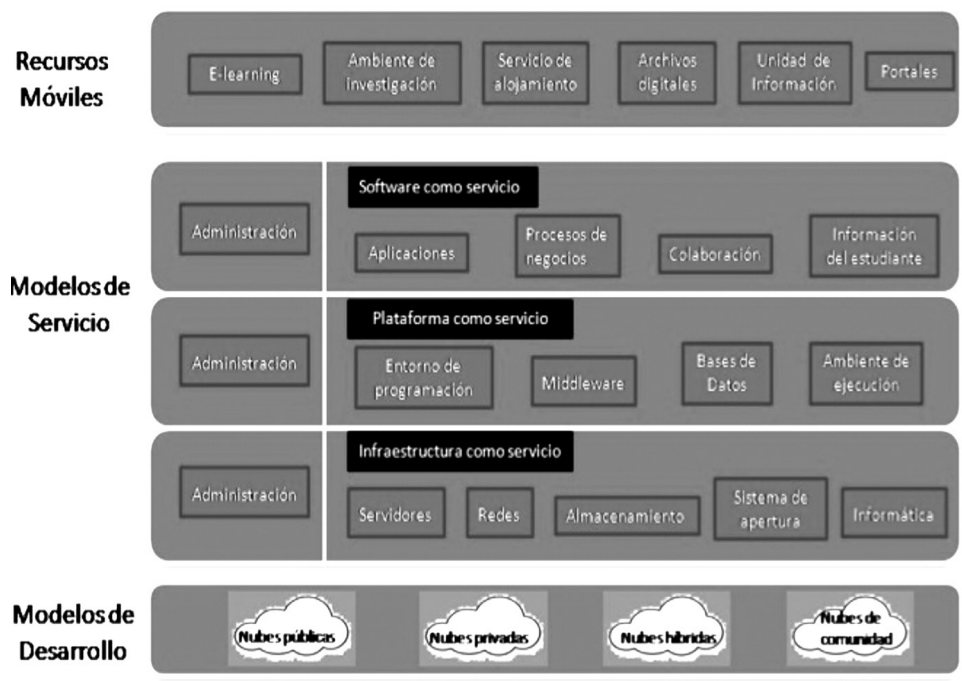

Figura 2. Arquitectura de la nube para la universidad ${ }^{4}$ Fuente: adaptación propia basada en Mircea y Andreescu (2011)

Añade la información de acuerdo a necesidades de información

Administrar el contenido de la información almacenada

Facilita procesos de comunicación/colaboración/y retroalimentación

Facilita la consulta de contenidos

Figura 3. Método para tener acceso y poder modificar informaciones en la nube Fuente: adaptación propia basada en Mircea y Andreescu (2011)

4 El middleware es un software que asiste a una aplicación para interactuar o comunicarse con otras aplicaciones, software, hardware y/o sistemas operativos 
El uso exitoso de la nube informática en la educación superior supone la existencia de tres elementos clave: la virtualización, la inteligencia de la red y un ecosistema robusto (Bozzelli, 2009). Estos tres elementos ofrecen la base para la obtención de la eficiencia operativa, la seguridad, la actividad de continuidad, escalabilidad e interoperabilidad.

\section{ESTRATEGIA DE ADOPCIÓN DE LA NUBE PARA UNA UNIVERSIDAD}

La migración hacia la nube necesita una estrategia bien definida que brinde soporte a las capacidades requeridas por la nube informática de acuerdo con las tecnologías de información existentes. El éxito de la implementación de la estrategia depende de la existencia de una arquitectura orientada a los servicios en el ámbito de la institución. Es importante resaltar que el análisis se debe hacer desde un punto de vista integral, recopilando información sobre necesidades específicas de cada una de las áreas o departamentos que integran la universidad.

Para tener éxito, la estrategia de implementación de la nube debe estar alineada con la estrategia de la universidad (Figura 4). A partir de las recientes investigaciones relacionadas con la transición a la nube informática y la experiencia de las unidades de información en su uso, se sugiere una estrategia de migración hacia la nube, formada por etapas que se aprecian en la Figura 4.

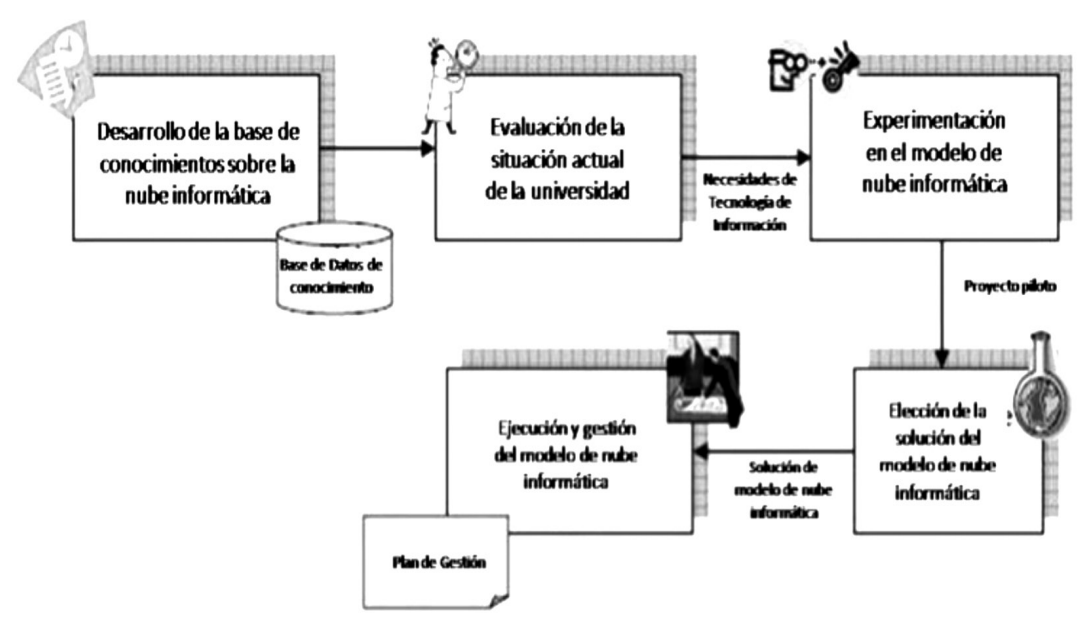

Figura 4. Estrategia de la nube en las unidades de información 
El primer paso consiste en desarrollar la base de conocimientos mediante la participación en seminarios, conferencias, debates con los proveedores y la consulta de las investigaciones más recientes en el campo. El éxito de la fase depende de la asignación de recursos suficientes para la investigación, para la comprensión de cómo funciona la nube informática en diferentes estructuras de organización de la universidad se estudian los beneficios y los riesgos, se establecen políticas de uso y se obtiene información de las mejores prácticas de uso.

\section{Evaluación de la situación actual de la universidad}

Consiste en valorar la situación actual de la universidad desde el punto de vista de las necesidades de TI, la estructura y el uso. La primera etapa consta de la comprensión de la infraestructura de TI de la universidad, la arquitectura orientada a servicios representa la base para la comprensión de los datos, servicios, procesos y aplicaciones que pueden migrar o deben mantenerse dentro de la universidad, con el fin de observar la política de seguridad. Con respecto a las necesidades de TI, su estructura y su uso, el análisis puede comenzar desde las categorías de usuarios que interactúan con la infraestructura de TI (Figura 5) y sus necesidades. Es muy importante plantearse una serie de preguntas y llevar a cabo un diagnóstico de la situación que se presenta al almacenar documentos de archivo digital, debido a que se tiene que conocer quién, cómo y qué tipo de funciones se llevan a cabo en las universidades, debido a que lo anterior nos brinda el tipo de documentos y expedientes que se generan en las mismas; además de con qué fines y por cuanto tiempo requieren ser preservados.

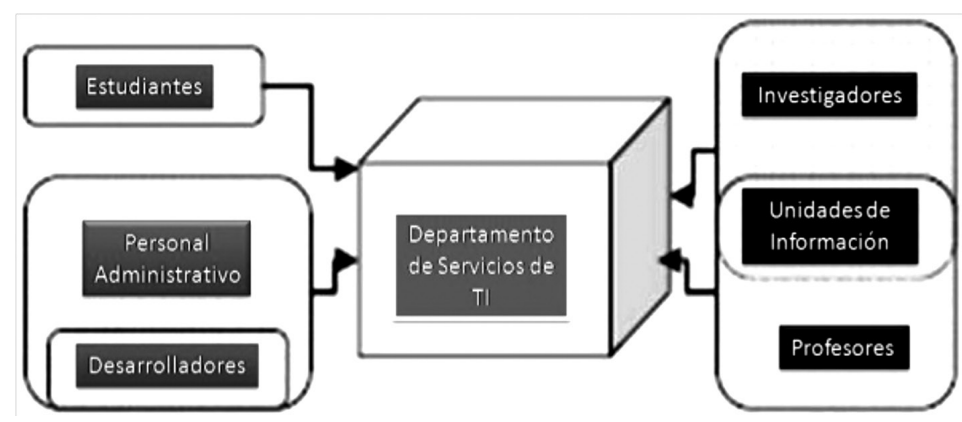

Figura 5. Estructura simplificada de los principales usuarios de servicios TI en una universidad Fuente: adaptación propia basada en Mircea y Andreescu (2011) 
En las universidades se generan documentos todos los días, según las funciones y tareas que se llevan a cabo en todas las áreas, con diversas finalidades. Por ello y siguiendo las indicaciones del Consejo Internacional de Archivos, tomaremos la siguiente definición con respecto al documento: "Información registrada, producida o recibida en el inicio, en el proceso o en la resolución de una actividad de una institución o de un particular, y que consta del contenido, del contexto y de la estructura suficiente para proveer a la actividad de valor probatorio" (Marcos, 1999).

Hay que aclarar que el contexto se refiere a los diferentes aspectos que afectan al documento, por ejemplo, quién firma, la relación que tiene con otros documentos (del mismo expediente o de otros), la función que ejerce, entre muchos otros aspectos.

Se deben considerar las siguientes características del documento digital si queremos almacenarlos en la nube:

- El contenido se registra de manera que para leerlo es preciso disponer de una computadora o un aparato lector adecuado, mientras que los documentos tradicionales de archivo pueden ser leídos directamente por el ojo humano.

- El contenido del documento digital puede ser separado del medio en el que se registró originalmente. El motivo por el que se cambia de soporte es la obsolescencia de los sistemas por el avance de la tecnología. Es fundamental asegurar que la información no varíe, independientemente de los respaldos que se lleven a cabo, evitando manipulaciones que pongan en duda su autenticidad y su fiabilidad, características fundamentales en la documentación de archivo. Por ello, al almacenarla en la nube se tendrá que poner una gran atención en la seguridad de la información.

- La estructura física de un documento digital no es considerada y no es válida para determinar la autenticidad de la información que contiene, ya que puede sufrir cambios al pasar de una versión a otra; en cambio, existe una estructura lógica que permite identificar cada documento y representar los elementos de su estructura interna (campos, márgenes, párrafos...). Se trata de la estructura que el creador dio al documento y que deberá ser la que aparezca en la pantalla del usuario.

- La preservación de estos nuevos documentos también difiere, ya que el tiempo que se estima que se puede conservar varía entre cinco y 30 años, según la versión y el espacio digital donde se almacena. A esto se 
suma la rapidez con que se vuelven obsoletos los sistemas, y el consiguiente volcado que hay que hacer de unos soportes a otros para poder seguir accediendo a la información. Por tanto, el modo de preservar estos documentos conlleva la copia constante de unos sistemas a otros.

Ahora bien, para su selección se tendrá que considerar los criterios siguientes:

- Origen y procedencia. Aquí tendremos que conocer la situación y las condiciones que originaron la producción del documento; es fundamental estar al tanto de la importancia y funciones de la institución creadora.

- El contenido es relevante, por lo tanto, se tendrá que analizar la información que brinda el documento y ver si refleja las actividades principales de la institución.

- Considerar el autor, la institución, la fecha y el lugar es importante para asegurar la autenticidad de algunos documentos. Además, el criterio de autenticidad tiene que centrarse en la validez jurídica del documento.

\section{Experimentación en el modelo de nube informática}

La transición a la nube se puede lograr poco a poco, a partir de pruebas en un proyecto piloto y luego exteriorizar las aplicaciones elegidas para la nube. El primer paso consiste en la solución de algunos de los objetivos, como el desarrollo y las pruebas de medio ambiente o almacenar algunos datos en la nube, el siguiente paso puede consistir en la elaboración diaria de las operaciones internas, abordando al mismo tiempo los componentes de nube pública y privada con el fin de asegurar las políticas de seguridad y protección. El mantenimiento de bajos costos para el uso de la solución debe ser tomado en cuenta de forma permanente. Recordemos que para almacenar documentos de archivo en el entorno de la nube se requiere de la autentificación, esta medida de control de acceso permitirá proteger los datos para asegurar su validez. Para poder realizar lo anterior se ha utilizado con mucha frecuencia un sistema de autentificación llamado Trusted Platform Module (TPM). Éste no sólo considera el nombre de usuario y la contraseña sino que es un estándar sobre la autorización de usuarios y otras herramientas de seguridad de comunicación en tiempo real entre el proveedor y el cliente. 


\section{La elección del modelo de nube informática}

El primer paso consiste en identificar los datos (Figura 6) y aplicaciones, funciones y principales procesos dentro de la universidad, que pueden ser agrupados de acuerdo a las tres grandes categorías de actividades universitarias: docencia, investigación y apoyo administrativo para las dos primeras. Además, es fundamental conocer lo que está surgiendo en el mercado en relación a la nube informática, ya que de eso depende el tipo de servicios y gestiones que se pueden llevar a cabo. Se tendrá que investigar sobre los nuevos avances e implementaciones tecnológicas, por ejemplo, la elección de la nube es una tema clave para las instituciones, por eso algunas empresas como Red Hat han trabajado desde hace bastante tiempo en propuestas y tecnologías que se adapten a las necesidades de los clientes. Esta nueva oferta de productos apuesta a la Open HybridCloud (nube híbrida abierta), que combina infraestructura pública y privada con el objetivo de que el usuario final pueda obtener y mover servicios entre distintos tipos de nubes, sin estar atados a un proveedor o formato tecnológico. Con lo anterior se puede asegurar que ya se pueden elegir algunas características o elementos para la nube de distintos proveedores. El paquete ownCloud, por su parte, ofrece a todos los usuarios los siguientes servicios: almacenamiento de información, uso compartido de archivos con diferentes usuarios y no usuarios, reproducción de música, galerías de imágenes, calendario/agenda de contactos, visor de archivos planos y editor de textos. Permite además las funciones más usuales, como el backup, la sincronización de archivos entre varios dispositivos y el uso de la nube con la tecnología WebDAV (Santamaría Buitrago, Rodríguez Amézquita y Molina Sosa, 2014). Conocer las ofertas tendrá como finalidad conseguir la eficiencia y el ahorro de recursos mediante la mejora de los subsistemas de clasificación, conservación y recuperación en la nube informática, sin dejar de lado aspectos de metadatos, servicios y necesidades de los usuarios, entre muchos otros componentes.

Para poder elegir lo mejor de los distintos tipos de nubes se tendrán que conocer muy bien las ventajas e inconvenientes que han de tenerse en cuenta en relación a las necesidades que pretendemos cubrir con el uso de la nube. 


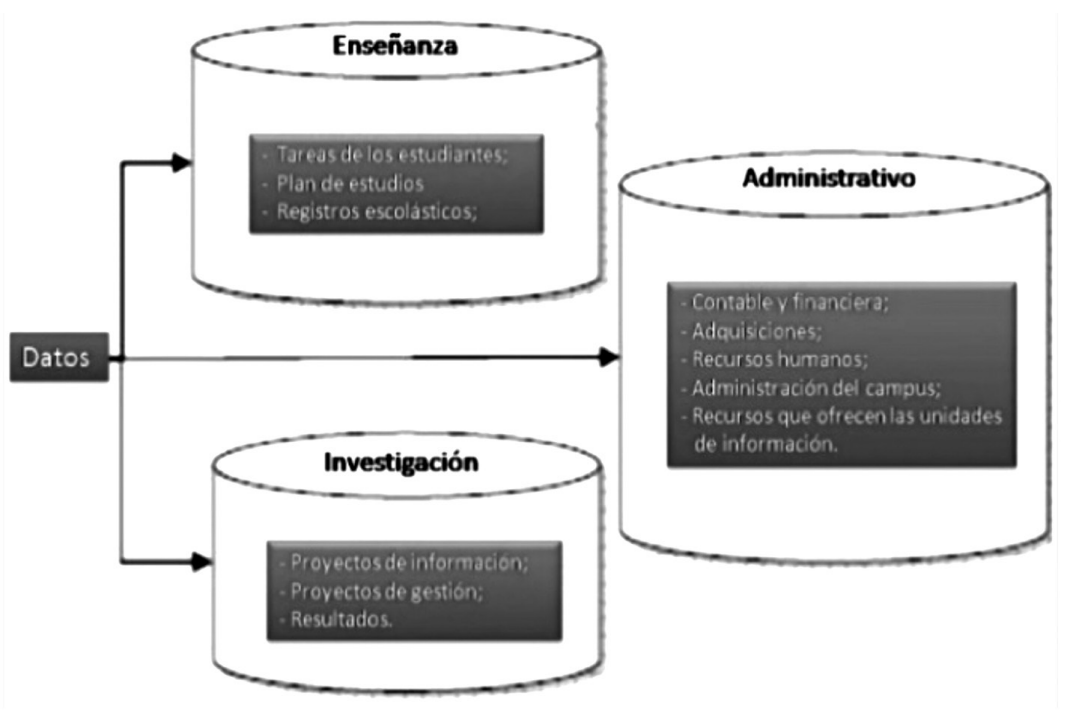

Figura 6. Principales datos de una universidad Fuente: adaptación propia basada en Mircea y Andreescu (2011)

\section{Ejecución y gestión del modelo de nube informática}

La solución puede implementarse en fases iterativas mediante una transición continua de los datos, servicios y procesos hacia la nube. Se lleva a cabo sobre la base de algunas de las evaluaciones continuas de los beneficios de la tecnología de nube sobre la universidad. Al mismo tiempo, la implementación supone el establecimiento de un programa flexible de gestión de riesgos, probando el rendimiento de la solución y gestión de la aplicación.

La migración de los datos, servicios y procesos hacia la plataforma en la nube debe hacerse sobre la base de algunos modelos y estrategias bien definidas. Cada modelo de migración supone objetivos específicos de acuerdo con la política de la organización, el control y la seguridad de la información. La migración de datos debe ser realizada para mantener un equilibrio óptimo entre la precisión de los datos, la velocidad de la migración, el tiempo que no funcione y los costos mínimos. A nivel de organización debe haber un modelo de gestión que incluya las políticas relativas a la seguridad, la gestión de las aplicaciones y la infraestructura, la gestión de los riesgos y la evaluación continua de la solución de la nube informática (Mircea, Ghilic-Micu y Stoica, 2011). Una gestión eficiente es esencial para cualquier programa de gestión 
de calidad. Es compatible con la seguridad proactiva de la calidad mediante la medición y la mejora de los procesos, procedimientos y servicios que realiza. Se tendrá que considerar en esta fase la idea de que los documentos de archivo universitario tendrán una intervención de todos los actores que integran su comunidad. Además se deben considerar los documentos digitales de archivo durante todo su ciclo de vida: creación, mantenimiento, utilización y disposición final.

\section{Conclusiones}

A pesar de sus críticos y los inconvenientes, la nube informática está aquí para quedarse. La situación económica actual obligará a las organizaciones a considerar la adopción de una solución de nube. Las universidades han comenzado a adherirse a esta iniciativa y hay pruebas de la disminución significativa de los gastos debido a la implementación de soluciones en la nube.

Es importante identificar las particularidades de uso de la nube informática para almacenar y gestionar todos los documentos que se generan en las instituciones de educación superior y desprender de ahí el uso que se le pueda dar en las unidades de información, teniendo en cuenta los riesgos y los beneficios de la arquitectura de nube y analizar estrategias de adopción de la nube adecuadas para las universidades.

Almacenar documentos digitales de archivo en la nube puede ser un beneficio para las unidades de información con o sin recursos económicos. Hoy en día los beneficios de la nube son numerosos, como es la flexibilidad, la agilidad para consulta de información y la rentabilidad. Las unidades de información, conscientes del gran crecimiento que se tiene en el manejo de información contenida en documentos de archivo, deben aprovechar las bondades que brinda el emplear el almacenamiento de información en la nube.

En otros ámbitos y con el transcurrir del tiempo, el uso de la nube se está convirtiendo en algo popular y confiable. Los servicios de la nube han demostrado ser una solución eficaz para todo tipo de unidad de información que maneje grandes cantidades de información y en específico documentos digitales de archivo, así como también para sus usuarios.

No debemos olvidar que este sistema de almacenamiento trabaja según la filosofía de cliente-servidor, donde el almacenamiento de la información en la nube se refiere a un entorno en el que los equipos cliente se conectan a varios servidores remotos, responsables de la gestión y tratamiento de datos, y estos a su vez, en conjunto (la red de servidores remotos), conforman el entorno de la nube. 
Con la administración de uso de la nube se mantiene en tiempo real el flujo de información que se requiere para una empresa, hospital, unidad de información, etc. Hoy en día se cree que los servidores de la nube son una mejor alternativa a los servidores físicos.

Unidades de información con experiencia en este rubro consideran el almacenamiento en la nube como una alternativa viable a los servidores físicos, con lo que han obtenido agilidad, escalabilidad y flexibilidad en el flujo de información. Otro factor que se ha presentado para mejorar el uso de la nube es que los servidores de la nube son a menudo PC virtuales, debido a que la configuración de un PC virtual no es difícil y reduce el costo en un grado sustancial.

Es importante resaltar que para iniciar un proyecto de este tipo debe existir conocimiento sobre lo que representa el uso virtual de la información. No obstante los avances de la tecnología, algunos responsables de las unidades de información no incursionan en este tipo de proyectos por no tener conocimiento de lo que implica el uso de la nube informática.

$\mathrm{Al}$ almacenar documentos digitales de archivo se deben considerar, además de las cuestiones de infraestructura y administración de la nube informática, aspectos particulares que tienen que ver con el uso y manejo de los documentos digitales (uso jurídico-administrativo). Se debe poner especial interés en conservar ante todo los valores primarios y secundarios de los mismos. El valor primario se refiere a cuestiones que reseñan lo legal, administrativo, fiscal, contable y técnico, su valor secundario se refiere a lo cultural, histórico, de investigación o científico.

$\mathrm{Al}$ organizar y almacenar documentos de archivos en la nube debemos tener una cultura patrimonial bien cimentada, debido a que debemos entender que los documentos dan fe de los acontecimientos y tareas realizadas dentro de cualquier institución y que por ello al preservarlos estaremos conservando la memoria de lo que acontece cada día en materia administrativa, histórica, financiera o social, entre muchos otros aspectos, y que al seleccionar y conservar los documentos de archivo estaremos asegurando que las generaciones futuras podrán hacer uso de esos documentos con diferentes finalidades de información. 


\section{REFERENCIAS}

"Algunos consejos antes de subir archivos a la nube". 2013. Revista Cloud Computing. http://www.revistacloudcomputing.com/2013/04/algunos-consejos-antes-de-subir-archivos-a-la-nube/\#sthash.DxmZOWol.dpuf

Arrabales, R. 2013. "Nube privada o pública: flexibilidad vs seguridad”. BSP Review: A Business, Software And Processes Magazine 4. http://sapreviews.com/pdf/04/ bspreviews04_MAY2013_Nube_privada_o_publica.pdf

Bozzelli, T. 2009. "Will the Public Sector Cloud Deliver Value? Powering the Cloud Infrastructure”. CISCO. http://www.cisco.com/web/strategy/docs/gov/2009_ cloud_public_sector_tbozelli.pdf

Cuadros, A. 2013. Computación en la Nube. http://tecnato.com/wp-content/ uploads/2013/03/nube.jpg

Faci Lacasta, M. 1995. "Metodología y aplicaciones de los principios de identificación y valoración en España: Resultados Obtenidos", en Entre historiadores y archivistas: el dilema de la valoración documental, 65-75. México: AGN.

Flantrmsky, H. 2012. "La computación en nube y el cambio del universo informático". Pensamiento y cultura del Universo Informático 15 (1): 88-93. http://pensamientoycultura.unisabana.edu.co/index.php/pyc/article/view/2216/2802

Katz, R., P. Goldstein y R. Yanosky. 2010. "Cloud Computing in Higher Education”. EDUCAUSE. http://net.educause.edu/section_params/conf/CCW10/highered.pdf

Kaufman, L. M. 2009. "Data Security in the World of Cloud Computing". IEEE Security and Privacy Magazine 7 (4): 61-64. http://redisboyacallanos.org/documents/ MemoriasCIIIS2014.pdf\#page $=162$

Marcos, M. C. 1999. "Los archivos en la era digital”. El profesional de la información. http://www.elprofesionaldelainformacion.com/contenidos/1999/junio/los_archivos_en_la_era_digital.html

McIrvine, S. 2010. "Making Cloud Computing Safe Trust, Security, Resiliency, Availability and Complexity”. IBM Corporation. https:/www950.ibm.com/events/wwe/ grp/grp004.nsf/vLookupPDFs/Security\%20\&\%20Cloud\%20PCTY\%20\%20 McIrvine/\$9ile/Security\%20\&\%20Cloud\%20PCTY\%20-\%20McIrvine.pdf

Mircea, M., B. Ghilic-Micu y M. Stoica. 2011. "Combining Business Intelligence with Cloud Computing to Delivery Agility in Actual Economy”. Journal of Economic Computation and Economic Cybernetics Studies. http://pits-bi-real-time.googlecode.com/svn/trunk/Tesis/Lecturas/Real-time/COMBINING\%20 BUSINESS\%20INTELLIGENCE\%20WITH\%20CLOUD\%20COMPUTING\%20 TO\%20DELIVERY\%20AGILITY\%20IN\%20ACTUAL\%20ECONOMY.pdf

Mircea, M. y A. I. Andreescu. 2011. "Using Cloud Computing in Higher Education: A Strategy to Improve Agility in the Current Financial Crisis". Communications of the IBIMA. DOI: $10.5171 / 2011.875547$

NIST (National Institute of Standards and Technology). 2011. http://csrc.nist.gov/ groups/SNS/cloud-computing

Patterson, D. 2010. "Cloud Computing and the RAD Lab”. UC Berkeley, Reliable adaptive Distributed Systems Lab. http://www.mvdirona.com/jrh/TalksAndPapers/PattersonMSCloudComputingRADLab.pdf 
Pérez Quesada, T. 1995. "La experiencia cubana en la valoración y selección documental”, en Entre historiadores y archivistas: el dilema de la valoración documental, 59-64. México: AGN.

Peset Reig, M. 1989. "Los archivos universitarios: su contenido y sus posibilidades", en Estudio en recuerdo de la profesora Sylvia Romeu Alfaro, t. II, 759-772. Valencia: Universidad.

SafeNet. 2010. "SafeNet Solutions Secure Cloud Computing Deployments". SafeNet Inc.http://www.safenetinc.com/About_SafeNet/

Santamaría Buitrago, F. A., J. C. Rodríguez Amézquita y F. Molina Sosa. 2014. "Administración y Configuración de una Nube Privada Segura y Personalizada para Pymes”, en Proceeding CIIIS 2014, 165-178. http://redisboyacallanos.org/documents/MemoriasCIIIS2014.pdf\#page $=162$

Para citar este texto:

Cabral Vargas, Brenda. 2018. "Consideraciones para el almacenamiento de archivos digitales en la nube informática en bibliotecas universitarias”. Investigación Bibliotecológica: archivonomía, bibliotecología e información 32 (74): 55-75.

http://dx.doi.org/10.22201/iibi.24488321xe.2018.74.57909 